\title{
Dispersive IR-Spectroscopy for harsh environment based on thermopile elements realized with TPL640 (thermopile line array)
}

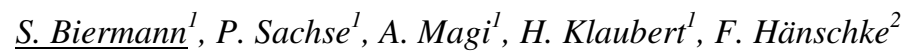 \\ ${ }^{1}$ Micro-Hybrid Electronic GmbH, Heinrich-Hertz-Str. 8, 07629 Hermsdorf, Germany \\ infrared@micro-hybrid.de \\ ${ }^{2}$ Institut für Photonische Technologien e.V., Albert-Einstein-Straße 9, Germany
}

\begin{abstract}
:
The condition monitoring of technical fluids and gases is a fundamental key challenge for the success of ongoing efforts to save valuable fossil resources like oil and gas and to protect our environment in the future. It's a prerequisite for an optimized utilization of raw materials and in this context essential for a cost efficient production of goods in the entire industry. So more and more often there is a substantial need for robust, cost efficient and also portable measurement devices for condition monitoring. Capitalizing on the advantages of the 64- pixel line array TPL640 it's possible to build up dispersive infrared spectrometers which have the desired properties.

The 64- pixel line thermopile array detects infrared radiation and converts it to an analog voltage signal. Such important parameters like high sensitivity and specific detectivity of each pixel are top values in comparison to other similar products on the market. The excellent spectral range of sensitivity from $0.7-25 \mu \mathrm{m}$ allows various applications. Special absorption layers can be established according to the specific requirements of the application.

For realization of complete spectrometer systems IR-sources are a necessary key component. Micro-Hybrid Electronic GmbH developed a range of different packages -JSIR350- based on patented NAC (nano amorphous carbon) MEMS chips which are aligned to various applications. The sources ideally meet requirements for pulsed operations within a range of $1 \ldots 100 \mathrm{~Hz}$. Excellent properties regarding highest emissivity due to very high membrane temperature and special developed packaging technologies for robust and hermetically sealed housings are key parameters of these components.
\end{abstract}

Key words: infrared line array, infrared spectroscopy, condition monitoring, infrared component, infrared source

\section{Outline}

There are different material analysis methods based on infrared spectroscopy generally known. In the first capture a short overview is given which shows the advantages und disadvantages of some selected principles. In correspondence with the view of robust spectroscopy devices based on line arrays an overview of different kinds of array technologies with their properties is presented. In additional the special properties of the TPL640, the packaging technology, the technical parameters, an example of application and an outlook on further developments are described. Then a short introduction of the developed infrared source JSIR350 as a key component in spectroscopy devices is posed. Finally, the special properties of the components TPL640 and JSIR350 are summarized. This shows the possibility to construct infrared spectroscopy devices get highest resolution for material analysis at lowest form factor with these integrated components.

\section{Principles of IR spectroscopy}

First a comparison of the following common methods is described:

1. FTIR - Fourier Transform Infrared Spectroscopy

2. NDIR - Non Dispersive Infrared Spectroscopy

3. Dispersive Infrared Spectroscopy with monochromator and single detector

4. Dispersive Infrared Spectroscopy with line array

1. Nowadays the FTIR spectroscopy is a well-known and common method. Since computer engineering allows to calculate the spectrum of an interferogram by the Fast Fourier Transmission algorithm, the FTIR spectrometers have achieved a wide distribution and is right now standard laboratory equipment for material analyses and optical characterization. The advantages are high resolution, high signal to noise ratio and high speed. Nevertheless it shows drawbacks like high costs, large shape and high weight and the sensibility of 
the equipment caused by the movable parts like the interferometer of the equipment.

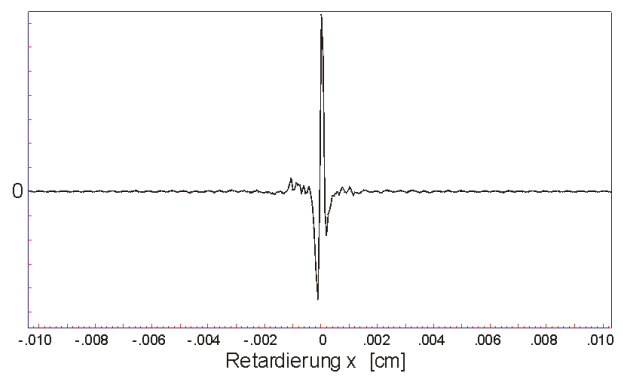

Fig. 1 Interferogram of a FTIR spectrometer

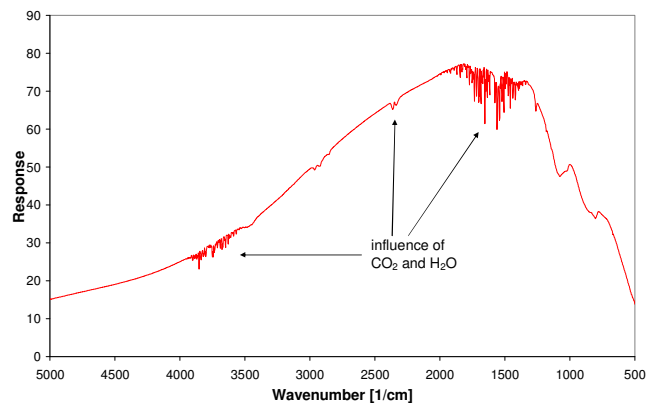

Fig. 2 Background Spectrum of a FTIR spectrometer on air

2. The NDIR, the Non Dispersive Infrared Spectroscopy is another well-known and common method. It is especially used when e.g. the concentration of a gas is to be determined in a known gas-mixture. Therefore you have to know what kind of gas or liquid you want to determine. The measurement cell is aligned with this special application and cannot be used for others. The application fields are gas and liquid measurements for environmental technologies, industry and healthcare. Advantageous features are the robust design, short response time and the low costs. The disadvantages are the low spectral resolution and selectivity. Only discrete analyses are possible.

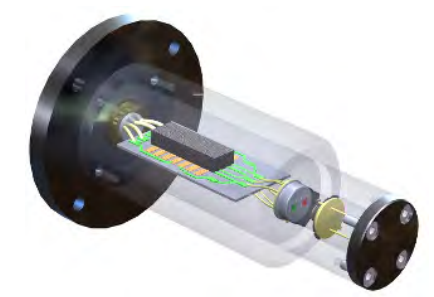

Fig. 3 Principle of a NDIR gas measuring chamber

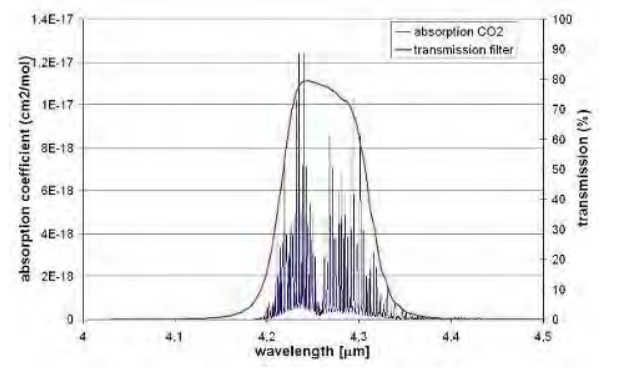

Fig. 4 Absorption of $\mathrm{CO} 2$ in dependence on the wavelength and transmission curves of the used filters
3. The dispersive infrared spectroscopy performed by a monochromator and a single detector is also a well known method. The infrared light of a broad band source is separated into its spectral parts by a monochromator. The dispersive element is commonly a movable grid. At the output of the monochromator a slit which only a narrow wavelength range passes is arranged. By moving the grid different wavelengths pass the slit. The instrument is used as a part of standard laboratory equipment for material analyses and optical characterization. The advantages are the high resolution and the adjustable monochromatic beam. The existing disadvantages are the low speed, the large shape and heavy weight, the mechanical sensitivity because of the movable parts like the grating and the high cost of such equipment. More often this method will be displaced by FTIR devices.

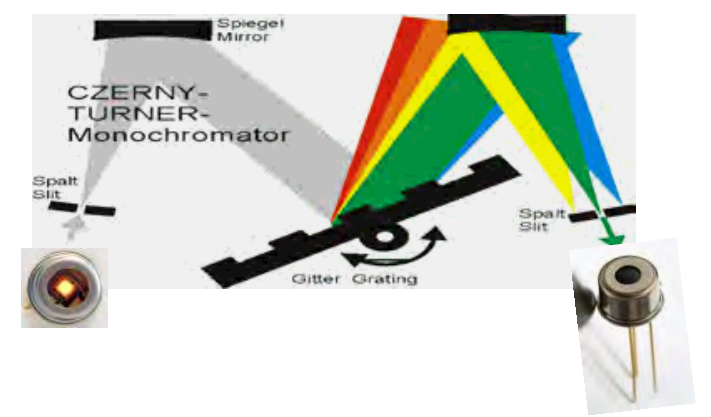

Fig. 5 Principle of a dispersive spectrometer with monochromator and single detector

4. The dispersive infrared spectroscopy with a line array is quite similar in comparison to the last one. But now we have a fixed grid and at the output of the device a line array which includes a lot of single pixels is arranged instead of a single detector. Each pixel sees a different wavelength of the IR-light at the same time. Because of the advantages the robust design (no movable parts) and the high speed the method is best suitable for handheld application of fluid and gas analysis. The spectral resolution depends on the number of pixels of the infrared line array and is lower in comparison to FTIR or dispersive spectrometers with a monochromator. However, in most cases this is entirely sufficient for the special spectral range of the application.

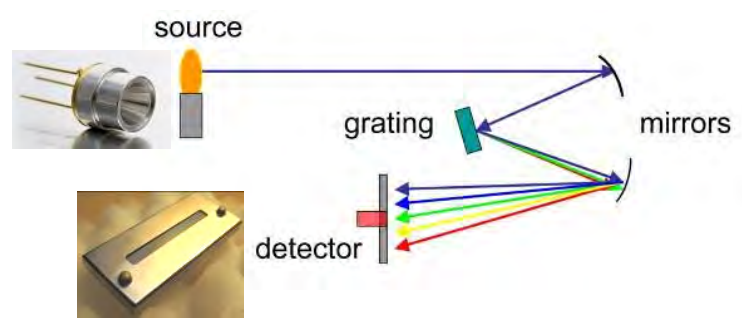

Fig. 6 Principle of a dispersive spectrometer with a line array 


\section{Infrared line arrays}

In the following passage an overview of typical available infrared array types based on thermal detectors is given.

The underlying principle of bolometer arrays is based on temperature dependent electrical resistance. The incoming radiation generates a temperature rise of the bolometer pixel and so a resistance change. This technology is best suitable for thermal imaging but not appropriate for spectroscopy application because of the non-linearity of the output signal.

A pyroelectrical device generates an electrical charge over the temperature rise, based on incoming radiation. The value depends on the radiation power. Because of the discharge over the surface of pyroelectrical elements the irradiation needs to be chopped to generate a continuous signal level. The minimum signal resolution can be described by the parameter NEP - noise equivalent power.

$$
N E P=\frac{\text { NoiseVoltage } / \sqrt{H z}}{R v}\left[\frac{W}{\sqrt{H z}}\right] \text { (1) }
$$

It describes the incoming radiation power of the detector which generates a signal level equal to the internal noise voltage. So a lower NEP level shows a better performance of the device. The NEP level of typical pyroelectric line arrays is at about $1.5 \mathrm{nW} / \mathrm{Hz}^{1 / 2}$.

Line arrays based on thermopiles generate a DC output voltage according to the incoming radiation power. The output level depends beside on geometrical parameters and the number of the thermocouples at one pixel as well as on the type of the thermopiles. That means the kind of the thermoelectrically materials of the thermocouples. Typical polysilicon based thermopile arrays show an NEP level of about $0.6 \mathrm{nW} / \mathrm{Hz}^{1 / 2}$ related to a sensitivity of $60 \mathrm{~V} / \mathrm{W}$ and thermopile resistance of $80 \mathrm{kOhms}$.

In contrast, the TPL640 which is based on BiSb/Sb thermocouples reaches an excellent NEP level of $0.08 \mathrm{nW} / \mathrm{Hz}^{1 / 2}$. This is achieved by a low thermopile resistance of $8.5 \mathrm{kOhms}$ and a high sensitivity of $155 \mathrm{~V} / \mathrm{W}$. The better performance of the $\mathrm{BiSb} / \mathrm{Sb}$ combination relates to the higher thermoelectric power of this material system. Based on this high performance level of the NEP a significant higher resolution of the measurement application is achievable compared to any other line array types.

\section{Features of TPL640}

The product features of the infrared line array TPL640 are summarized in the following notes:

- Highest sensitivity of $155 \mathrm{~V} / \mathrm{W}$ given by the $\mathrm{BiSb} / \mathrm{Sb}$ thermocouples.
- Excellent signal to noise ratio with an NEP of only $0.08 \mathrm{nW} / \mathrm{Hz}^{1 / 2}$.

- Line array chip contains 64 independent thermopile pixels.

- Very low cross talk of the line chip given by thermal separated pixels. There are slits in the membrane between each pixel.

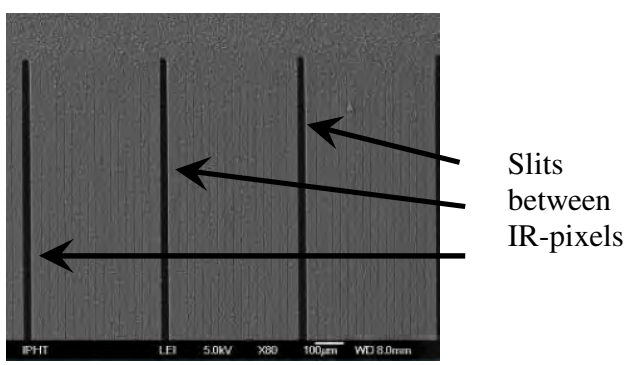

Fig. 7 Extract of the line array chip

- Good uniformity of sensitivity below than $5 \%$ and a deviation of resistance between adjacent pixel less than 1/30 guaranteed by the single line chip design.

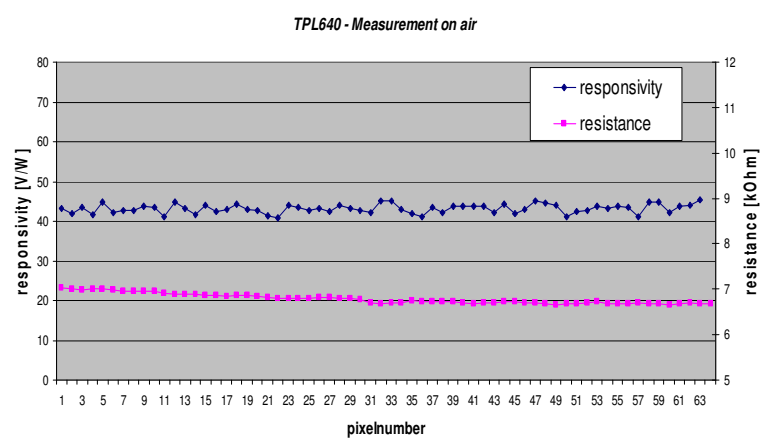

Fig. 8 Measurement diagram of sensitivity and resistance of each pixel

- The package is filled with inert gas xenon to achieve high sensitivity and long-term reliability.

- Broadband absorbance characteristic of the line array.

\section{Special properties of TPL640}

A special key property represents the wide spectral sensitivity of each pixel between $0.7 \mu \mathrm{m}$ and $25 \mu \mathrm{m}$. This characteristic is based on the used $\mathrm{Ag}$ - soot absorption layer, which has a very good absorption coefficient over a wide spectral range. This Ag soot generates a large surface. On the other hand the weight of this absorption layer is extremely low and does not adversely affect the time constant of the pixel. In thermopile detectors a common used interference absorber has an optimized characteristic for special spectral ranges. Because of this unequal behavior they are not suitable for spectroscopy applications such as in the line array. 


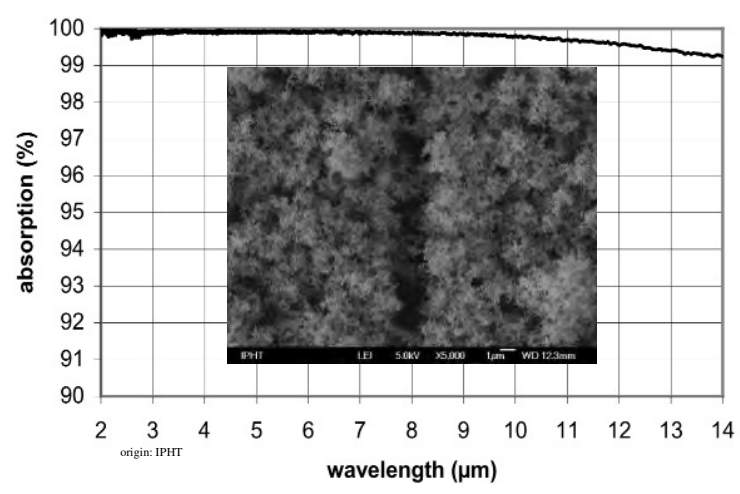

Fig. 9 Spectral emission coefficient of the Ag-soot absorber + REM picture of the surface

The line array chip is assembled in a high precision packaging and housing to adapt an external optics in an exact position to the internal pixels. This is given by an accurate position of the array chip in the packaging. We developed a special construction of the bathtub header and the lid with internal and external position pins. By an interlocking and automate - based assembly process we achieve a tolerance between the pins outside on the lid and the sensitive element of $+/-0.025 \mathrm{~mm}$.

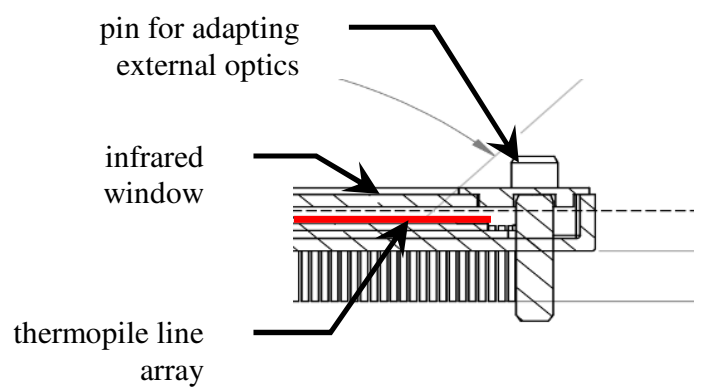

Fig. 10 Construction of housing TPL640

Moreover the packaging technologies solve the problems with different thermal expansion coefficients of the different materials by a special equalizing layer. The figure shows an explosion drawing of the header assembly.

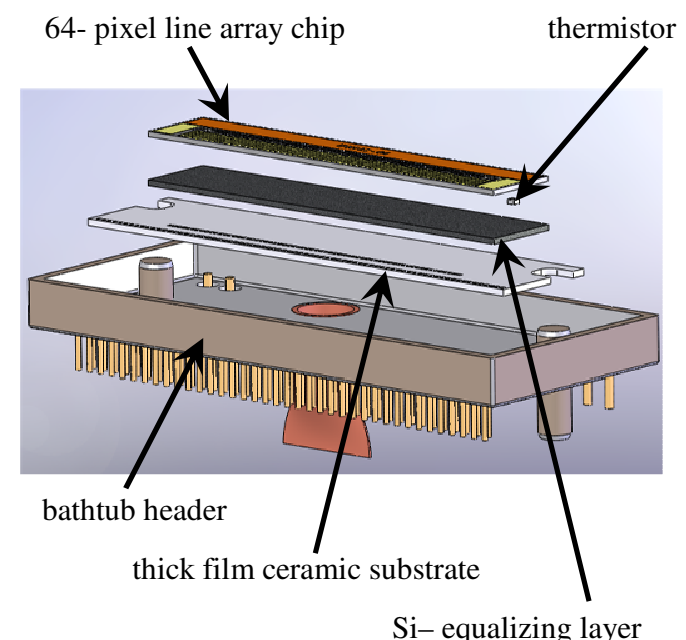

Fig. 11 Explosion drawing of header assembly
From the bottom to the top is shown:

- The bathtub header

- contains the pinch tube to allow bakeout and gas backfill processes

- The thick film ceramic substrate for

○ cable run between IR-line and header pins

- positive-fit connection to the header

○ position marks for chip assembly

- $\quad$ Si- equalizing layer

- reduces mechanical stress by different thermal coefficients

- Thermistor

- measures the temperature of the Si-bulk of the line array

- 64-pixel line array chip

$\circ$ measures the incoming infrared radiation

\section{Packaging technology of TPL640}

To ensure a high process reliability the header assembly is realized by an automatic die bonder VICO 520M. With the support of the vision based pick and place system the line chip is assembled in a high accuracy. The electrical connection between line chip and header is set up by an automatic wire bonder Bondjet BJ820. The wedge - wedge ultrasonic bonding technology with Al wire with a diameter of $32 \mu \mathrm{m}$ is used. The last assembly step is the sealing process which is realized by a special vacuum / bake out equipment. The TPL640 is fixed by the copper tube of the bathtub in a quick fastener. We generate a dry internal atmosphere by several evacuation, bake-out and flush cycles with dry nitrogen. With this equipment we can generate a vacuum up to $1 \times 10^{-5}$ mbar. Finally the housing is backfilled with $\mathrm{Xe}$ and sealed by squeezing the pinch tube of the header with a special gripper tool.

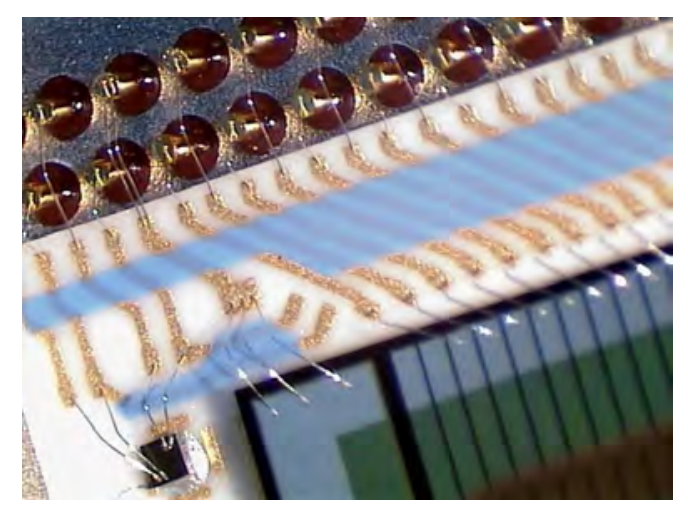

Fig. 12 Extract view of TPL640 packaging

\section{Application reference}

Here we present an application reference of our TPL640. It's used in a handheld spectrometer device called FluidScan® $Q 1000$ from Spectro Inc. 
employed for fluid analysis. It provides the possibility for a quick check of the conditions of:

- hydraulic fluids,

- engine oils,

- turbine oils and

- gear oils

for degradation and the presence of contaminants.

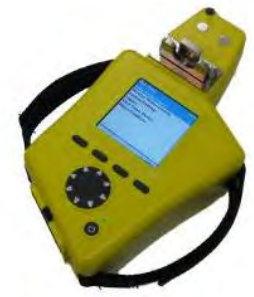

origin: www.spectroinc.com/productsfluidscan.htm

Fig. 13 FluidScan ${ }^{\circledR}$ Q1000 from Spectro Inc

\section{Technical parameters TPL640}

In the following table the most important technical parameters of the line array are shown.

\begin{tabular}{|c|c|c|c|}
\hline Pixel & \multicolumn{3}{|c|}{64} \\
\hline Active Area per Pixel & & $0.45 \times 1.5$ & $\mathrm{~mm}^{2}$ \\
\hline Pitch & & 0.5 & $\mathrm{~mm}$ \\
\hline Number of Thermocouples per Pixel & & 30 & \\
\hline Pixel Resistance (at $25^{\circ} \mathrm{C}$ ) & & $8.5 \pm 3.5$ & $\mathrm{k} \Omega$ \\
\hline $\begin{array}{l}\text { Relative Deviation of Resistance } \\
\text { Between Adjacent Pixel }\end{array}$ & & $<1 / 30$ & \\
\hline $\begin{array}{l}\text { Temperature Coefficient Resistance } \\
\text { of Thermopile }^{1}\end{array}$ & typ. & -0.4 & $\% / K$ \\
\hline $\begin{array}{l}\text { Temperature Coefficient of } \\
\text { Sensitivity }^{1}\end{array}$ & & -0.6 & $\% / \mathrm{K}$ \\
\hline Noise Voltage (at $25^{\circ} \mathrm{C}$ ) & typ. & 12 & $\mathrm{nV} / \mathrm{Hz}^{1 / 2}$ \\
\hline Filling Gas & & Xenon & \\
\hline $\begin{array}{l}\text { DC Output Voltage (at } 38 \mu \mathrm{W} / \mathrm{mm}^{2} \text {, } \\
\text { at } 25^{\circ} \mathrm{C} \text { ) }\end{array}$ & typ. & 4.0 & $\mathrm{mV}$ \\
\hline $\begin{array}{l}\text { DC Sensitivity (at } 38 \mu \mathrm{W} / \mathrm{mm}^{2} \text {, at } \\
\left.25^{\circ} \mathrm{C}\right)^{2}\end{array}$ & typ. & 155 & $\mathrm{~V} / \mathrm{W}$ \\
\hline Time Constant $\mathrm{t}_{(0.63 \%)^{2}}$ & typ. & 110 & $\mathrm{~ms}$ \\
\hline $\begin{array}{l}\text { Specific Detectivity } \\
D^{*}(500 \mathrm{~K} \text { black body, DC, at } 25\end{array}$ & typ. & $1.08 \times 10^{9}$ & $\mathrm{cmHz}^{1 / 2} / \mathrm{W}$ \\
\hline Noise Equivalent Power (at $\left.25^{\circ} \mathrm{C}\right)^{2}$ & typ. & 0.08 & $\mathrm{nW} / \mathrm{Hz}^{1 / 2}$ \\
\hline Crosstalk Between Adjacent Pixels ${ }^{2}$ & typ. & 25 & $\%$ \\
\hline Filter $^{3}$ & typ. & $\begin{array}{r}\text { AR coated } \\
\text { Ge }\end{array}$ & $\begin{array}{l}\text { Average } \\
\% \mathrm{~T}: \geq 90 \% \\
\text { from } 2- \\
16 \mu \mathrm{m}\end{array}$ \\
\hline Working Temperature & & $-20 \ldots+70$ & ${ }^{\circ} \mathrm{C}$ \\
\hline
\end{tabular}

\section{Application board TPL640}

For a first start-up process of the line array we developed an easy application board. This contains:

An analog signal preprocessing with an analog multiplexer to combine the 64 single pixels and a programmable low noise amplifier.

A digital processing unit with a 16 bit A/D converter and a microcontroller for processing and conditioning of the digital signals and a serial RS232 interface.
And a PC based LabView software shows an easy graphical indication and gives the possibility to record the signal curve.

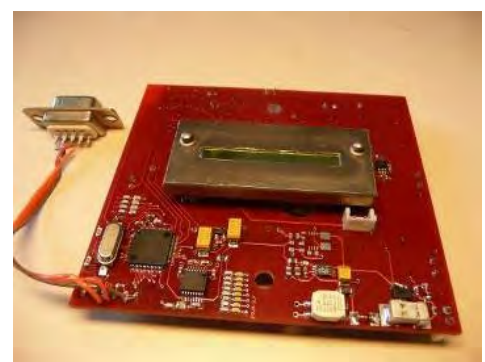

Fig. 14 Application board TPL640

\section{Outlook}

In order to allow optimal utilization of the parameters of the infrared line array chip a stable vacuum atmosphere is necessary. Providing this condition we can expect an increase of the sensitivity by factor 1.6 and the same way a reduction of the cross talk between adjacent pixels to $0.4 \%$ in comparison to the $\mathrm{Xe}$ atmosphere. To achieve this goal the development of a suitable getter technology was started in a new government funded project.

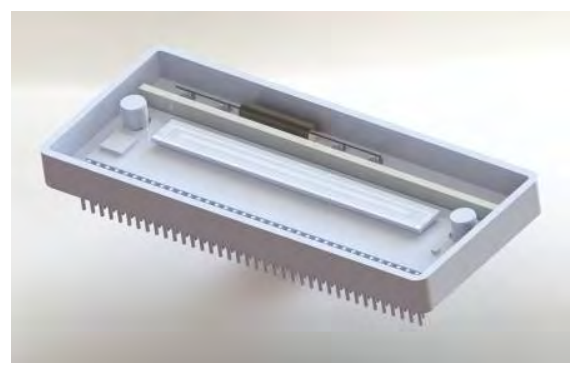

Fig. 15 TPL640 with integrated getter and heat shield

A further possibility to increase the sensitivity of the line array is the implementation of a new thermal electrical material system with a better efficiency such as $\mathrm{BiSb} / \mathrm{BiSbTe}$. We have realized this new technology at a prototype status with our four channel detector in TO39 housing successfully. A signal level enhancement by factor 3 could be achieved.

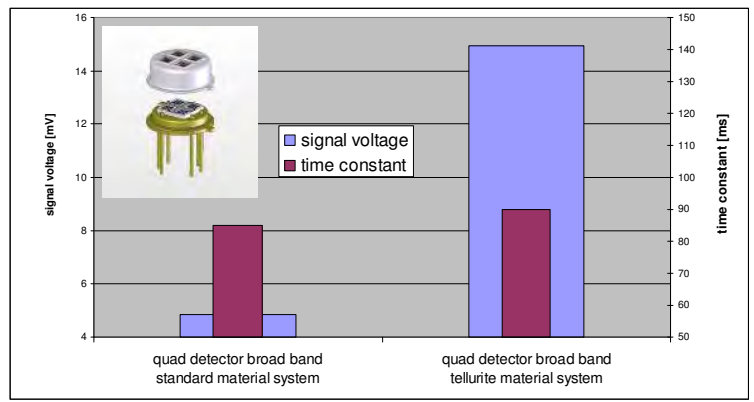

Fig. 16 Diagram of increase of the sensitivity by using BiSb/BiSbTe material system

The increase of the number of pixels up to 256 can be realized by a complete new chip production 
technology based on the Surface Micromachining technology. The pixels will be realized as freehanging membranes at the top of a $\mathrm{Si}$ wafer. The absorber will be realized as a combination of $\lambda / 4$ absorber and Ag-soot surface. This new technology development will be accomplished by a government funded project with the IPHT - Jena.
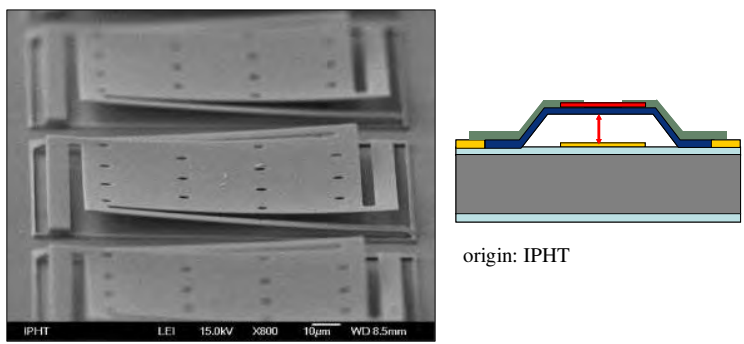

origin: IPHT

Fig. 17 Free-hanging membranes produced by Surface Micromachining technology

\section{Infrared source JSIR 350}

The infrared source is a complementary key component for the detector line array for the realization of complete spectrometer systems. Micro-Hybrid Electronic GmbH developed a range of different high performance infrared sources based on an MEMS infrared emitter chip with an active surface made of a nano amorphous carbon (NAC) layer system. The emitter chip consists of a $\mathrm{Si}$ frame, a membrane which contains the active material - a nano amorphous carbon layer system and the bond pads. The membrane of the chip is realized by a back etched process. Thin film processing is done by Magnetron Sputtering.

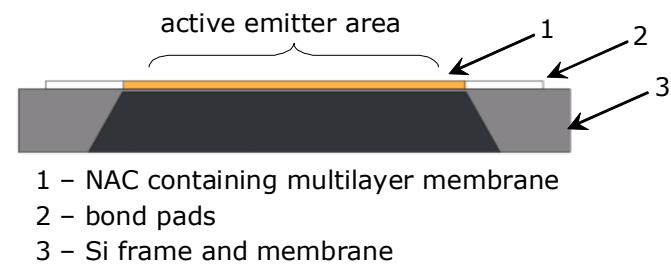

Fig. 18 Structure of MEMS emitter chip

The product features of our infrared source are:

- fast electrical modulation possible up to $100 \mathrm{~Hz} \rightarrow$ no mechanical chopping needed

- $\quad$ high temperature of active area causes high irradiation power

- $600{ }^{\circ} \mathrm{C}$ at nominal power $0.8 \mathrm{~W}$

- $700{ }^{\circ} \mathrm{C}$ at max. power $1.0 \mathrm{~W}$

- radiation near the black body curve

- lifetime up to 100,000 hours

- different robust designs $\rightarrow$ TO39; TO46 and SMD packages
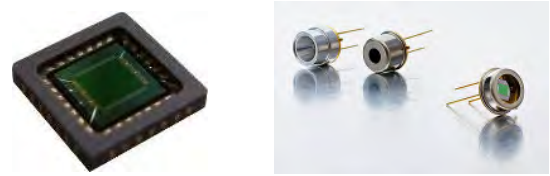

Fig. 19 Different packages of ir-emitter
- customized filter and reflector available

- real hermetically sealed housing and window

- $\quad$ various emitter chips with different active sizes available e.g. $0.5 \times 0.5 \mathrm{~mm}^{2}$ and $2.2 \times 2.2 \mathrm{~mm}^{2}$

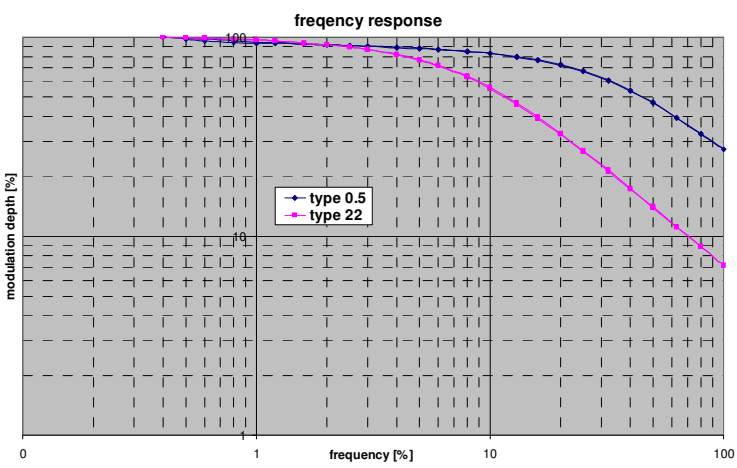

Fig. 20 Frequency response of different infrared emitter types

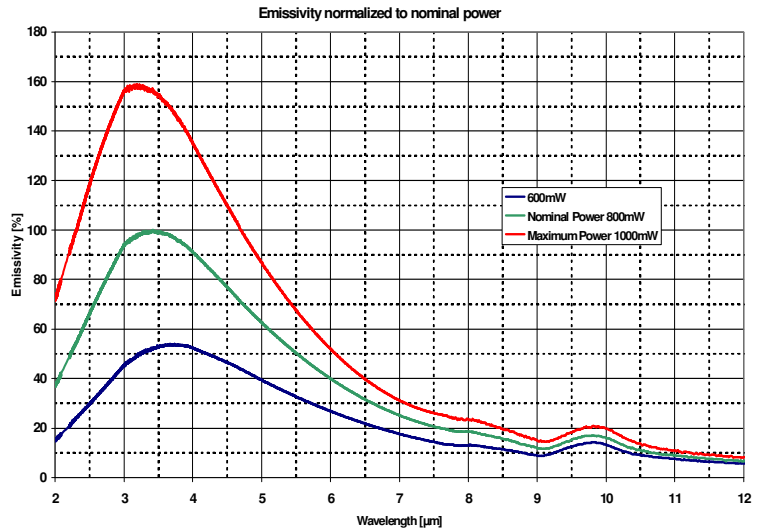

Fig. 21 Spectral emission curve at different input power levels

\section{Summary}

For condition analysis in harsh environments the dispersive Infrared Spectroscopy with line arrays is the method of choice. The TPL640 is an outstanding device for spectroscopy applications because of:

- $\quad$ superior signal resolution $\rightarrow$ low NEP

- high overall sensitivity

- $\quad$ robust and precise packaging

The infrared source is a key component in spectroscopy devices and the JSIR350 exhibits excellent features for that application such as:

- high irradiation output $\rightarrow$ membrane temperature up to $700{ }^{\circ} \mathrm{C}$

- $\quad$ high frequency response up to $100 \mathrm{~Hz}$

- robust hermetically sealed packaging

\section{References}

[1] Micro-Hybrid Electronic GmbH, Datasheet TPL640, http://www.micro-hybrid.de/fileadmin/user/bilderalt/Datenblaetter/Thermopiles/MH_TPL640.pdf; $01 / 31 / 13$ 